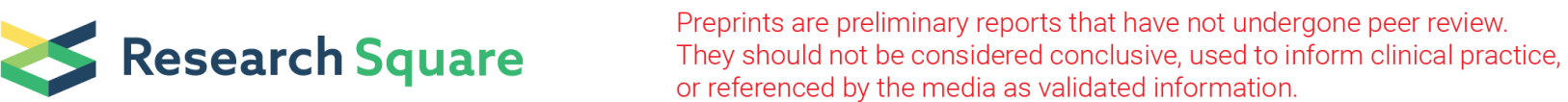

\section{Has-miR-875-5p Inhibits Tumorigenesis By Targeting USF2 Via TGF- $\beta$ Signaling Pathway in Gastric Cancer}

Shenshuo Gao (D2415695124@qq.com )

Shandong University Affiliated Hospital: Shandong Provincial Hospital https://orcid.org/0000-00016883-4827

Zhikai Zhang

Shandong University Affiliated Hospital: Shandong Provincial Hospital

Xubin Wang

Shandong Provincial Hospital

Yan Ma

Shandong Provincial Hospital

Chensheng Li

Shandong Provincial Hospital

Hongjun Liu

Shandong Provincial Hospital

Changqing Jing

Shandong Provincial Hospital

Leping Li

Shandong Provincial Hospital

Xiaobo Guo

Shandong University Affiliated Hospital: Shandong Provincial Hospital

\section{Research Article}

Keywords: miR-875-5p, USF2, Gastric Cancer, TGF- $\beta$

Posted Date: November 1st, 2021

DOI: https://doi.org/10.21203/rs.3.rs-885737/v2

License: (c) (1) This work is licensed under a Creative Commons Attribution 4.0 International License.

Read Full License 


\section{Abstract}

Background: Gastric cancer (GC) is one of the most common malignancies, and more and more evdiences show that the pathogenesis is regulated by various miRNAs. In this study, we investigated the role of miR-875 in GC.

Methods: The expression of miR-875-5p was detected in human GC specimens and cell lines by miRNA RT-PCR. The effect of miR-875-5p on GC proliferation was determined by CCK-8 proliferation assay and EDU assay. Migration and invasion were examined by transwell migration and invasion assay and wound healing assay. The interaction between miR-875-5p and its target gene USF2 was verified by a dual luciferase reporter assay. The effects of miR-875-5p in vivo were studied in xenograft nude mice models. Related proteins were detected by Western blot.

Results: The results showed that miR-875-5p inhibited the proliferation, migration and invasion of gastric cancer cells in vitro, and inhibited tumorigenesis in vivo. USF2 proved to be a direct target of miR-875-5p. Knockdown of USF2 partially counteracts the effects of miR-875-5p inhibitors. Overexpression of miR875-5p can inhibit proliferation, migration, and invasion through the TGF- $\beta$ signaling pathway by downregulation of USF2 in GC, providing a new research direction for the diagnosis and targeted therapy of GC.

Conclusions: MiR-875-5p can inhibited the progression of GC by directly targeting USF2 and negatively regulating TGF- $\beta$ signaling pathway. In the future, miR-875-5p is expected to be used as a potential therapeutic target for GC therapy.

\section{Background}

Gastric cancer is one of the most common malignant tumors of the digestive tract due to excessive proliferation of gastric epithelial cells. Worldwide, gastric cancer ranks fourth in morbidity and second in mortality[1]. Due to the lack of effective biomarkers for early diagnosis, gastric cancer patients often develop to an advanced stage when they are diagnosed. and their 5-year survival rate is less than $30 \%[2]$. Early diagnosis of GC can avoid this deterioration and improve survival in patients with GC. In order to improve the prognosis of GC patients, more effective biomarkers for early diagnosis are needed. Therefore, there is an urgent need to understand the basic mechanism of CG tumorigenesis and development and to find effective biological targets. Although changes in oncogenes and tumor suppressor genes have been reported in gastric cancer, the pathogenesis of gastric cancer, the complex molecular mechanisms and signal transduction pathways involved in the progression and development of gastric cancer have not been fully studied, which limits effective biological targets and clinical treatment[3]. Therefore, the exact molecular mechanisms of gastric cancer pathogenesis still need to be fully clarified.

MicroRNAs (miRNAs) are a class of small, highly conserved, non-coding single-stranded RNA molecules encoded by endogenous genes, 18 - 22 nucleotides in length, that directly target the 3 ' untranslated 
region ( $3^{\prime}-U T R$ ) of genes by binding to certain sequence specific sites[4]. The silencing complex induced by miRNA prevents the translation or direct degradation of mRNA, resulting in a decrease in the expression of these genes [5]. There are various regulations between miRNA and mRNA, affecting epigenetics, RNA stability and translation[6]. MiRNA plays an important role in cell proliferation, metastasis, differentiation, apoptosis and development[7]. It has been shown that miRNAs are abnormally expressed in cancer tissues compared to normal tissues[8, 9]. There are still many unknown details about the role of miRNAs in human cancer and further research is needed.

MiR-875-5p is dysregulated in many diseases, including gestational diabetes mellitus[10], liver fibrosis[11], lung cancer[12], esophageal cancer[13], hepatocellular carcinoma[14], prostate cancer[15], colorectal carcinoma[16] and thyroid cancer[17]. These results suggest that miR-875-5p plays a role in the occurrence and development of tumors, in the previous study, we found that the expression of miR-875$5 p$ was decreased in gastric cancer[18], but its role in gastric cancer remains unclear. This study aims to explore the effect of miR-875-5p on gastric cancer and its potential mechanism.

Upstream stimulator (USF) was first identified in HeLa nuclear extract because of its ability to bind to elements in the major late promoter of adenovirus (E-box) and stimulate gene transcription[19, 20]. Two USF peptides with apparent molecular weights of 43 and $44 \mathrm{kDa}$ were obtained from HeLa cells, called USF1 and USF2, respectively[21, 22]. USF-2 is basic helix-loop -helix (BHLH) transcription factors encoded by heterodimeric and overlapping genes[23]. Isotypes produced by splicing show different transcriptional activities in certain promoter environments[24, 25]. Although it is widely believed that USF2 expression and relative abundance vary [26], it interact synergistically with other factors in tissue and stimulusspecific transcriptional regulation[27,28]. Studies have shown that USF2 plays a promoting role in the development of breast cancer, colorectal cancer and lung cancer[29-32].

This study aims to explore the biological function of miR-875-5p in gastric cancer and its potential mechanism. We demonstrate for the first time that miR-875-5p directly targets and regulates the 3'-UTR of human USF2 mRNA, which is upregulated in many cancers, including gastric cancer. Here, we report that miR-875-5p is indeed inhibited in primary gastric cancer compared to matched adjacent normal gastric tissues and find that the $3^{\prime}-$ UTR of human USF2 mRNA is actually the target of miR-875-5p. Further experiments showed that miR-875-5p inhibited the proliferation, migration, and invasion of gastric cancer cell lines by directly binding to the 3 ' untranslated region of USF2 and inhibiting USF2 expression at the protein level, then affects the TGF- $\beta$ signaling pathway. Further experiments showed that miR-875-5p inhibited the expression of USF2 at the protein level by directly binding to the 3 'untranslated region of USF2, and thus affected the TGF- $\beta$ signaling pathway, thus inhibiting the proliferation, migration and invasion of gastric cancer cell.

\section{Materials And Methods}

\section{Tissue samples}


Human GC tissues and paired adjacent normal tissues were collected from 36 patients with GC who underwent radical gastrectomy at the Department of General Surgery, Shandong Provincial Hospital Affiliated Cheeloo College of Medicine of Shandong University, China. After surgical excision, the sample was quickly frozen in liquid nitrogen for experiments. This study was approved by the ethics committee of Shandong Provincial Hospital. Written informed consent is signed prior to specimen collection. The collection of gastric cancer and paracancer tissue and its use for research purposes was approved by the Institutional Review Board of the Shandong Provincial Hospital affiliated to Shandong University, Shandong, China.

\section{Cell culture}

Six gastric cancer cell lines (BGC-823, HGC-27, MGC-803, SGC-7901, MKN-45, AGS) and GES-1 cell lines were purchased from Institute of Biochemistry and Cell Biology, Chinese Academy of Sciences (Shanghai, China). Cells were cultured in RPMI 1640 supplemented with 10\% fetal bovine serum (10\% FBS), 100 $\mathrm{U} / \mathrm{mL}$ penicillin, and $100 \mathrm{mg} / \mathrm{mL}$ streptomycin (Gibco, Grand Island, NY,USA) was incubated in moist air at $37^{\circ} \mathrm{C}$ and $5 \% \mathrm{CO} 2$.

\section{Cell transfection}

Hsa-miR-875-5p mimics and mimics negative control, hsa-miR-875-5p inhibitor and inhibitor negative control were purchased from Gemma. (Shanghai, China). At least 24 hours before transfection, the cells were cultured in complete medium without antibiotics. Cells were planted in six-well plates, when the cell fusion degree reached $50 \%-70 \%$, the cells were washed with $1 \times$ PBS (PH7.4), 50nM miR-875-5p mimics or miR-mimic NC and 100nM miR-875-5p inhibitors or miR-inhibitor NC were transfected into AGS and MKN45 cells through Lipofectamine ${ }^{\text {TM }} 2000$ (Invitrogen). Si-USF2 and si-NC were purchased from Genome (Shanghai, China) and transfected as above.

\section{RNA extraction and RT-qPCR}

Total RNA was extracted from GC tissues and cells using Trizol reagent (Takara, Japan) according to the manufacturer's instructions. The cDNA was synthesized using the Evo M-MLV RT Premix for qPCR reagent (AG, China). QRT-PCR was performed on Roche LightCycler 480 II fluorescent quantitative PCR instrument with qPCR SYBR Green Pro Taq HS Master Mix(AG). $\beta$-actin was used as the endogenous control. The primers used in this study are as follows: USF2 forward: 5'-AAAGGAGGGATCCTGTCCAA-3', USF2 reverse: 5'-CAGGGCGTTCTCATTCTTCA-3'; $\beta$-actin forward: 5'-GCATCGTCACTGGGGAC-3' and $\beta$ actin reverse: 5'-ACCTGG CCGTCAGGCAGCTC-3'. In addition, unchained curves were used to evaluate nonspecific amplification. QRT-PCR reaction procedures are as follows: $95^{\circ} \mathrm{C}$ for $30 \mathrm{~s}, 40$ cycles at $95^{\circ} \mathrm{C} 5$ $\mathrm{s}$ and $60^{\circ} \mathrm{C} 30 \mathrm{~s}$. All procedures were carried out in triplicate and relative expression was calculated by the $2^{-\triangle \Delta C T}$ method.

miRNA RT-PCR 
Total RNA was extracted as mentioned above. According to the manufacturer's instructions, using the Mir-X miRNA first-Strand Synthesis Kit (Takara, Japan), RNA $(2 \mu \mathrm{g})$ was converted to cDNA. PCR reaction was performed using TB Green ${ }^{\circledR}$ Premix Ex TaqTM II (Takara, Japan), and U6 was used as the endogenous control. The primers of Mir-875-5p and U6 were purchased from RiboBio (RiboBio Co., Ltd, Guangzhou, China), the primer sequences used in this study were as follows; has-miR-875-5p forward:5'GCGGGCGGTATACCTCAGTTTTAT-3', reverse 5'-ATCCAGTGCAGGGTCCGAGG-3'; U6 forward: 5'CTCGCTTCGGCAGCACA-3'; U6 reverse: 5'-AACGCTTCACGAATTTGCGT-3'. $2^{-\triangle \triangle C t}$ method was used to calculate the relative expression level. All procedures were also performed in triplicate.

\section{Protein extraction and Western blot}

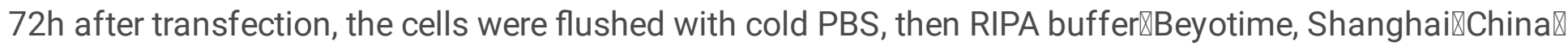
containing PMSF (SolarBio, Beijing, China) and phosphatase inhibitors (SolarBio, Beijing, China) was used for cracking. The protein concentration was calculated using the BCA Protein Assay Kit (Beyotime,

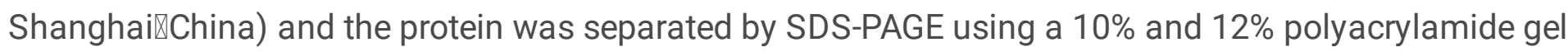
$(20 \mu \mathrm{g}$ per sample). Proteins are transferred to the immobilon-NC membrane by electrotransfer. The imblotted membrane was sealed in 5\% skimmed milk diluted with TBST, and then incubated with appropriate primary antibodies (anti-USF2, anti-p21, anti-p57, anti-E-cadherin, anti-Vimentin, anti-smad2, anti-phospho-smad2, anti-smad3, anti-phospho-smad3 and anti-GADPH obtained from CST) at $4^{\circ} \mathrm{C}$ for 12 h.

\section{Dual-luciferase reporting assay}

Dual luciferase assay was used to further verify the targeting relationship of miR-875-5p and USF2. Bioinformatics analysis predicted that the possible binding site of miR-875-5p was the 3 'UTR site 595601 of USF2 mRNA. AGS was grown in 1640 medium containing 10\% FBS and transfected with psicheck2-Husf2-3 'UTR reporter plasmid and human miR875-5p mimics using Lipofectamine ${ }^{\text {TM }} 2000$. The dual luciferase reporter gene system was used to detect whether human microRNAs bind and regulate USF2.

\section{CCK8 assay}

Cell growth was measured using cell proliferation reagent CCK-8 (MCE). Cells were inoculated into a 96well plate (Corning Costar, Corning, NY) at a concentration of $2.0 \times 10^{3}$ per well, as per manufacturer's instructions, and 10 $\mu$ LCCK8 was added to each well at harvest. One hour after CCK8 was added, cell viability was determined by measuring the absorbance of the transformed dye at 450nM.

\section{Edu experiment}

The transfected cells were seeded into a 96-well plate at a concentration of $1.0 \times 10^{4} /$ well. The EDU solution (Reagent A) was diluted with cell complete medium in a ratio of 1000:1 to prepare an appropriate

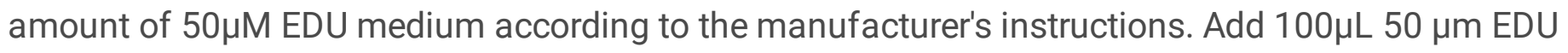


medium to each well and incubate for 2 hours, then discard the medium; PBS washed the cells twice, 5 minutes each time. Add $50 \mu \mathrm{l}$ cell fixation solution (PBS containing 4\% paraformaldehyde) to each well and incubate at room temperature for $30 \mathrm{~min}$, then discard the fixation solution; Add $50 \mu \mathrm{l} 2 \mathrm{mg} / \mathrm{mL}$ glycine to each well, and incubate on decolorizing shaker for $5 \mathrm{~min}$, then discard the glycine solution; Add $100 \mu$ l PBS to each well, wash with decolorizing shaker for $5 \mathrm{~min}$, discard PBS; Add $100 \mu$ l penetrant $(0.5 \%$ TritonX-100 PBS) to each well and incubate in decolorizing shaker for $10 \mathrm{~min}$. PBS cleaning once, 5 minutes; Add 1X Apollo ${ }^{\circledR}$ dyeing reaction solution of $100 \mu$ l to each well, and incubate for 30 min in dark, at room temperature, decolorizing shaker, then discard the dyeing reaction solution. Add $100 \mu$ penetrant (0.5\% TritonX-100 PBS) to decolorizing shaker, wash it 3 times, 10 min each time, discard penetrant; Add $100 \mu \mathrm{l}$ methanol to each well and clean it twice, 5 min each time; PBS cleaning once, 5 minutes each time; Add $100 \mu \mathrm{l} 1 \mathrm{X}$ Hoechst 33342 reaction solution to each well, and incubate in dark, at room temperature and decolorized shaker for $30 \mathrm{~min}$. Then discard the dyeing reaction solution. Add 100 $\mu$ PBS to each well

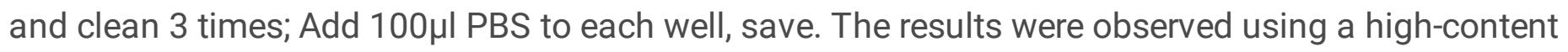
imaging system.

\section{Transwell migration/invasion assay}

MKN-45 and AGS cells grew to approximately 70\% confluence in RPMI 1640 containing $10 \%$ fetal bovine serum and then transfected. After 24 hours, the cells were digested by trypsin and then washed once with PBS. To measure cell migration, culture inserts with an $8 \mathrm{~mm}$ aperture (Transwell; CoStar, High Wycombe, UK) were placed in the holes of the 24-well plate and the upper and lower chambers were separated. In the lower chamber, add RPMI 1640 containing 20\% FBS, 600 $\mu$ l. Then, serum-free medium containing $5 \times 10^{4}$ cells was added to the upper compartment for migration assay, and $1 \times 10^{5}$ cells were used for matrix gel invasion assay. After incubation at $37^{\circ} \mathrm{C}$ and $5 \% \mathrm{CO} 2$ for $24 \mathrm{~h}$, the cells in the upper compartment were removed with a cotton swab. The cells that invaded the base membrane of the inserts were fixed in $100 \%$ methanol for $10 \mathrm{~min}$, stained with $1 \%$ crystal violet for $20 \mathrm{~min}$, rinsed in PBS, and examined microscopically. Each experiment was performed at least three times.

\section{Wound healing assay}

To evaluate the motility of GC cells. A total of $1 \times 10^{6}$ cells/Wells were inoculated in a 6-well plate and cultured overnight, then transfected with miR-875-5p mimics or NC, miR-875-5p inhibitor or NC and siUSF2 or NC. Eight hours later, the confluent cell monolayers were scraped with a sterile pipette head and the plates were washed twice with PBS and cultured for 24 hours before adding fresh serum-free medium. An image of the plate is taken under a microscope. The clearance size was analyzed with Image J software.

\section{Tumorigenesis in nude mice}

BALB/c nude mice (male, 4-6 weeks old, 16-20 g) were purchased from Beijing Vital River Laboratory Animal Technology Co., Ltd (Beijing, China). All animal experiments were conducted in accordance with the Guidelines for the Care and Use of Laboratory Animals of Provincial Hospital Affiliated to Shandong 
University. MiR-875-5p NC and miR-875-5p mimics were transfected into MKN-45 cells, and $5 \times 10^{5} \mathrm{MKN}-$ 45 cells in logarithmic growth phase were suspended in $100 \mu \mathrm{L}$ phosphate buffer, and then seeded subcutaneously into the right axillary of nude mice. The experiments were divided into two groups on average (miR-875-5p NC group and miR-875-5p mimics group, $n=6$ ), tumor size was monitored by measuring length $(L)$ and width $(W)$ with a vernier caliper every 4 days, and volume was calculated using the following formula: $\left(L \times W^{2}\right) / 2$. The mice were fed for 28 days. Tumors were sacrificed and collected. Tumor volume and weight was measured for analysis. Animal experiments conformed to the standards set by the Declaration of Helsinki, and were approved by the ethics review Committee of Shandong Provincial Hospital affiliated to Shandong University, Shandong, China.

\section{Immunohistochemical staining of xenograft tumors tissue}

Tumor sections were incubated overnight with commercial rabbit polyclonal antibodies against USF at 1:50 dilution at $4^{\circ} \mathrm{C}$. Then, the slices were diluted with horseradish peroxidase (HRP) antibody (1:100; Invitrogen, Thermo Fisher, US) was conjugated at room temperature for $2 \mathrm{~h}$ and then covered with DAB (SP kit (rabbit streptavidin-biotin method detection system), ZSGB-BIO, China). After rinsing the colored plates with water for a period of time, they were soaked in hematoxylin and dyed, then dehydrated and sealed. Subsequently, all fields of view were observed under a light microscope (Tissue FAXS Systems, Austria).

\section{Statistical Analysis}

The results are presented as means \pm SD. Statistical significance was measured by multiple comparisons using Student's t-test with a significance level of $p<0.05$.

\section{Results}

\section{miR-875-5p is down-regulated in human GC tissues and cell lines}

In order to confirm the abnormal expression of miR-875-5p in GC tissues, 36 pairs of GC tissues and adjacent normal tissues were collected and the relative expression of miR-875-5p was examined by miRNA RT-PCR. As shown in Figure.1A, the expression of miR-875-5p in human GC tissues was lower than that in paired adjacent tissues $(P<0.05)$. The expression of miR-875-5p was further examined by miRNA RT-PCR in normal gastric mucosal epithelial cells (GES-1) and GC cell lines (AGS, BGC-823, MKN45, HGC-27, MGC-803 and SGC-7901). As shown in Figure.1B, the expression of miR-875-5p in GC cell lines was lower than that in GES-1 cells $(P<0.05)$. These data suggest that miR-875-5p is downregulated in GC tissues and cell lines.

\section{miR-875-5p inhibits the proliferation of GC cell lines}

To investigate the potential role of miR-875-5p in GC tumorigenesis, miR-875-5p mimics and inhibitors were transfected into AGS and MKN-45 cells. Using CCK8 kit to measure cell growth in vitro, the results 
showed that compared with their negative control, overexpression or silencing of miR-875-5p significantly inhibited or promoted the proliferation of GC cells, respectively $(P<0.05$, Figure.2A). The EDU experiment further confirmed the role of miR-875-5p in inhibiting GC cells proliferation $(P<0.05$, Figure.2B). In addition, we also demonstrated that overexpression and silencing of miR-875-5p caused up-regulation or down-regulation of proliferation-related proteins P21 and P57 $(P<0.05$, Figure.2C). These results suggested that miR-875-5p could inhibit GC cell proliferation.

\section{miR-875-5p inhibits the migration and invasion of GC cell lines}

The inhibitory effect of miR-875-5p in GC cells was further investigated. The effect of miR-875-5p on GC cell migration and invasion was examined by cell migration and matrix invasion assay. As shown in Figure.3, the number of penetrating cells in miR-875-5p mimics group in AGS and MKN-45 cells was also reduced compared to the control group, while silencing of miR-875-5p showed the opposite effect in AGS and MKN-45 cells. In wound healing assay, overexpression of miR-875-5p reduced the migration rate of AGS and MKN-45 cells. In contrast, knockdown of miR-875-5p significantly accelerated AGS and MKN-45 cells migration (Figure.4A). In addition, we also demonstrated that overexpression and silencing of miR875-5p caused up-regulation or down-regulation of E-cadherinøVimentin proteins (Figure.4B). These results suggested that miR-875-5p inhibited cell migration and invasion in GC cells.

The effects of miR-875-5p on up-regulation and down-regulation of migration and invasion ability of GC cells were estimated by Transwell assay.

\section{USF2 is a predicted target of miR-875-5p}

The target of miR-875-5p was predicted by TargetScan (http://www.targetscan.org/vert_71/), miRmap (https://mirmap.ezlab.org/) and Pictar (https://pictar.mdc-berlin.de/). According to the predictions of three miRNA bioinformatics websites, we found that USF2, an oncogene in many malignancies, may be one of the target genes of miR-875-5p.

\section{USF2 is upregulated in human GC tissues}

To study the relationship between USF2 and GC, USF2 mRNA expression was analyzed in normal stomach specimens and gastric cancer tumor specimens in TCGA database using UALCAN nets. The results are shown in FIG. 5.a: Compared with normal tissues, USF2 mRNA level in GC tissues was significantly increased (normal $n=34$, LUAD $n=415, P<0.01$ ). In addition, Kaplan-Meier Plotter was used to detect the correlation between USF2 mRNA and prognosis in GS patients. We found that USF2 gene expression level significantly affected overall survival and survival in GC patients.

\section{The USF2 3'-UTR is a target of miR-875-5p}

Dual luciferase assay was used to verify the targeting relationship between miR-875-5p and USF2. Bioinformatics analysis predicted that the possible binding site of miR-875-5p was the 3 'UTR site 595601 of USF2 mRNA (Figure.5B). AGS was grown in 1640 medium containing 10\% FBS and transfected 
with psicheck2-Husf2-3 'UTR reporter plasmid and human miR-875-5p simulant or inhibitor using Lipofectamine ${ }^{\mathrm{Tm}} 2000$. After $48 \mathrm{~h}$ of culture, human microRNA was detected by the dual luciferase reporter gene system to determine whether the human microRNA binds and regulates USF2. Compared with the control group, luciferase activity was significantly reduced in AGS cells transfected with miR-875-5p mimics and psicheck2-USF2WT 3'-UTR. Luciferase activity in AGS cells transfected with miR-875-5p inhibitor and psicheck2-USF2WT 3'-UTR was significantly increased. The overexpression and knockdown of miR-875-5p did not affect luciferase activity in cells transfected with psicheck2-usf2 mut3 '-UTR (Figure.5B). After AGS and MKN-45 cells were transfected with miR-875-5p mimics, the mRNA and protein expression of USF2 were detected by RT-qPCR and Western Blot, and the mRNA and protein expression of USF2 were decreased. The results were reversed after transfection with miR-875-5p inhibitor. In addition, all of these results indicate that the $3^{\prime}-$ UTR of USF2 is targeted by miR-875-5p, and point mutations in this sequence cancel this interaction

\section{USF2 promote proliferation, migration, and invasion in GC cells}

In order to detect the potential effect of USF2 on the proliferation, migration and invasion of AGS and MKN-45 cells. AGS and MKN-45 cells were transfected with USF2 siRNA or negative control. CCK-8 and EDU experiments showed that USF2 knockdown significantly inhibited the proliferation of AGS and MKN45 cells (Figure.S9). We also demonstrated that knockdown of USF2 caused changes of proliferationassociated proteins (Figure.S9C). The wound healing experiment showed that knockdown of USF2 significantly inhibited the migration and invasion of AGS and MKN-45 cells (Figure.S10A). In addition, transwell assay showed that knockdown of USF2 significantly inhibited the migration and invasion of AGS and MKN-45 cells (Figure.S10B). We also demonstrated that knockdown of USF2 caused changes of migration and invasion related proteins (Figure.S10C).

\section{MiR-875-5p inhibits proliferation, migration, and invasion in GC cells by targeting USF2}

We demonstrated that miR-875-5p overexpression and USF2 knockdown inhibited proliferation, migration, and invasion in GC cells and inhibited USF2 protein expression through mRNA degradation. The results were opposite when miR-875-5p was silenced. In order to further confirm that miR-875-5p inhibited proliferation, migration and invasion in GC by regulating USF2. MKN-45 and AGS cells were cotransfected with miR-NC + si-NC, miR-875-5p inhibitor + si-NC, and miR-875-5pinhibitor + si-USF2. Through CCK8 assay and EDU assay, we found that down-regulation of USF2 in MKN-45 and AGS cells partially offset the effect of miR-875-5p silencing in proliferation (Figure.6). Proliferation-associated protein was detected by western blot (Figure.6C). Through transwell migration and invasion assay and wound healing assay, we found that down-regulation of USF2 in MKN-45 and AGS cells partially offset the effect of miR-875-5p silencing in migration and invasion (Figure.7). Migration and invasion related protein was detected by western blot (Figure.7C). These findings suggest that miR-875-5p inhibitions GC cell proliferation, migration, and invasion by directly targeting USF2.

\section{MiR-875-5p decreases the TGF- $\beta$ signaling pathway by downregulation of USF2}


To examine the mechanisms by which miR-875-5p and USF2 inhibit GC proliferation, migration, and invasion, we investigated whether miR-875-5p mediated these effects by affecting the TGF- $\beta$ signaling pathway. Western blotting was used to examine USF2 expression levels and downstream proteins Smad2, p-Smad2, Smad3 and p-Smad3. As shown in Figure.6C, the silencing of miR-875-5p showed a significant increase in p-Smad2 and p-Smad3 in GC cells compared to the control group. However, there was no significant difference in Smad2 and Smad3 expression. These effects can be partially counteracted by down-regulating USF2 expression in miR-875-5p inhibitor cells. These results suggest that USF2 is a downstream functional regulator of miR-875-5p, acting through the TGF- $\beta$ signaling pathway.

\section{MiR-875-5p suppresses the growth of GC in vivo}

To demonstrate the effect of miR-875-5p on tumor growth in vivo, miR-875-5p-mimics transfected cells were injected into the flanks of nude mice, and miR-NC transfected cells served as negative controls. As shown in Figure.8, compared with control group, the miR-875-5p mimics group showed a significant reduction in tumor volume and weight. In addition, immunohistochemistry was used to investigate the expression levels of USF2. Compared with the control group, USF2 protein levels was significantly reduced in miR-875-5p-mimics group (Figure.8B). In conclusion, miR-875-5p inhibited the growth of GC cell in vivo.

\section{Discussion}

Abnormal expression of miRNAs plays an important role in the occurrence and development of tumors[33-35]. Previous studies have shown that miRNA can bind to specific sites of certain sequences to directly target the 3'-UTR of genes and prevent their translation or direct degradation of mRNA, thus participating in the occurrence and development of tumors[9, 36, 37]. As a newly discovered tumor-related miRNA, the role of miR-875-5p in gastric cancer and the specific mechanism of its role are still unclear. In this study, we analyzed the expression level of miR-875-5p in GC tissues and normal tissue adjacent to cancer, gastric cancer cell lines and GES-1 cells, and predicted the target genes. In CCK8 and Edu experiments, the overexpression of miR-875-5p inhibited the proliferation of gastric cancer cells and affected the expression of proliferation-related proteins P21 and P57. The knockdown of miR-875-5p showed opposite results, indicating that miR-875-5p played an inhibitory role in the proliferation of gastric cancer cells. Tumor spread and metastasis are affected by the migration and invasion ability of tumor cells, both of which are inhibited by miR-875-5p in vitro. Transwell migration and invasion experiment showed that the overexpression of miR-875-5p inhibited the migration and invasion of gastric cancer cells. In addition, the expression of related proteins E-cadherin and Vimentin was affected, and the results were reversed when miR-875-5p was knocked down. Experiments in nude mice demonstrated that the overexpression of miR-875-5p inhibited the tumorigenicity of gastric cancer cells in vivo, and this series of experiments showed that miR-875-5p have an inhibitory effect on gastric cancer, suggesting that miR875-5p may be a potential therapeutic target for gastric cancer patients. Through a series of experiments, it was found that the overexpression of miR-875-5p inhibited the proliferation, migration, invasion and 
tumorigenicity of gastric cancer cells, suggesting that miR-875-5p could be a potential therapeutic target for GC patients. In addition, Western blotting and dual luciferase report analysis showed that USF2 is the target gene of miR-875-5p and is negatively correlated with miR-875-5p. Our study also showed that miR$875-5 p$ directly binds to the 3 '-UTR region of USF2 to inhibit its expression and down-regulate TGF- $\beta$ signaling pathway.

To elucidate the mechanism of the effect of miR-875-5p on proliferation, migration and invasion. Bioinformatics analysis predicted the putative target of miR-875-5p in GC cells. Among the candidate target genes, we selected USF2[32]. The upstream stimulatory factor(USF2) plays an important role in various cellular processes, especially in the genesis and progression of tumors[38-40]. However, the role of USF2 in different tumors is contradictory, suggesting that it is either a tumor promoter or an inhibitor[29-31, 41]. The role of USF2 in gastric cancer has not been studied. Therefore, it is very necessary to study its expression and role in gastric cancer. Compared with normal gastric tissue, the expression of USF2 in gastric cancer tissue was significantly increased. In CCK8 experiment and Edu experiment, the OD value and the proportion of proliferating cells in si-USF2 group were significantly decreased, indicating that the proliferation of gastric cancer cells would be inhibited after USF2 knockdown. Transwell migration and invasion experiment showed that the migration and invasion ability of gastric cancer cells would be inhibited after USF2 knockout. Dual luciferase experiments further confirmed that miR-875-5p could directly bind to the USF2 3'-UTR region and degrade USF2 mRNA. In addition, knockdown of USF2 partially offset the promoting effect of miR-875-5p silencing on proliferation, migration, and invasion. In conclusion, our results indicate that the inhibitory effect of miR$875-5 p$ in gastric cancer is mediated by down-regulation of USF2.

Previous studies have shown that TGF- $\beta$ signaling pathway is associated with tumor proliferation and metastasis[42-45]. USF2 has been reported to down-regulate Smurf1 and Smurf2, thereby regulating the TGF- $\beta$ pathway in breast cancer[30]. Therefore, we studied the TGF- $\beta$ pathway changes induced by miR875-5p expression in GC. Western Blot results showed that the expression of phosphor-SMAD2 and phosphor-SMAD3 increased after knockdown of miR-875-5p, while the expression of SMAD2 and SMAD3 remained unchanged and TGF- $\beta$ signaling pathway was activated. On this basis, the expression of phosphor-SMAD2 and phosphor-SMAD3 decreased slightly after knockdown of USF2. The expression of SMAD2 and SMAD3 remained unchanged. These results suggest that the decreased miR-875-5p can activate the TGF- $\beta$ signaling pathway by upregulation of USF2. These results suggest that the decreased miR-875-5p can activate the TGF- $\beta$ signaling pathway by upregulating USF2. In other words, miR-875-5p can be used as a negative regulator of TGF- $\beta$ pathway to inhibit the proliferation, migration and invasion of gastric cancer. However, it is not ruled out that miR-875-5p can also influence other signaling pathways to exert anti-cancer effects.

\section{Conclusion}

Our study showed that miR-875-5p was significantly down-regulated in gastric cancer tissues and gastric cancer cell lines. As a key inhibitor of the growth and metastasis of gastric cancer cells, miR-875-5p 
inhibited the progression of GC by directly targeting USF2 and negatively regulating TGF- $\beta$ signaling pathway. In the future, miR-875-5p is expected to be used as a potential therapeutic target for GC therapy.

\section{Abbreviations}

USF2: upstream stimulate factor2; miRNA: Micro RNA siRNA: Small interfering RNA; 3'-UTR: 3'untranslated region; qRT-PCR: Quantitative real-time PCR; FBS: Fetal bovine serum; EdU: 5-ethynyl-2'deoxyuridine: CCK-8: Cell Counting Kit-8; IHC: Immunohistochemistry; TGF- $\beta$ : transforming growth factor$\beta$

\section{Declarations}

\section{Ethics approval and consent to participate}

All institutional and national guidelines for the care and use of laboratory animals were followed.

\section{Consent for publication}

All authors have agreed with publishing this manuscript.

\section{Availability of data and materials}

The datasets used and/or analyzed during the current study are available from the corresponding author on reasonable request.

\section{Competing interests}

The authors declare no conflict of interest.

\section{Funding}

This work was supported in part by grants from the National Science Foundation of China (81101858,81672379), the Natural Science Foundation of Shandong Province of China (ZR2016HM16), Key Research Project from Shandong Science and Technology Commission (2011GGB14158).

\section{Author Contributions}

XG designed the project; SG contributed to perform in vitro and in vivo experiments and write article; ZZ, $X W$ and YM contributed to analyze experiment data; $C L, H L, C J$ and $L L$ provided advice and critical comments.

\section{Acknowledgements}

Not applicable. 


\section{References}

1. Sung H, Ferlay J, Siegel RL, Laversanne M, Soerjomataram I, Jemal A, Bray F. Global cancer statistics 2020: GLOBOCAN estimates of incidence and mortality worldwide for 36 cancers in 185 countries. CA Cancer J Clin 2021.

2. Cao W, Chen HD, Yu YW, Li N, Chen WQ. Changing profiles of cancer burden worldwide and in China: a secondary analysis of the global cancer statistics 2020. Chin Med J (Engl). 2021;134:783-91.

3. Suh YS, Na D, Lee JS, Chae J, Kim E, Jang G, Lee J, Min J, Ock CY, Kong SH, et al: Comprehensive Molecular Characterization of Adenocarcinoma of the Gastroesophageal Junction Between Esophageal and Gastric Adenocarcinomas. Ann Surg 2020.

4. Bartel DP. MicroRNAs: genomics, biogenesis, mechanism, and function. Cell. 2004;116:281-97.

5. Pu M, Chen J, Tao Z, Miao L, Qi X, Wang Y, Ren J. Regulatory network of miRNA on its target: coordination between transcriptional and post-transcriptional regulation of gene expression. Cell Mol Life Sci. 2019;76:441-51.

6. Zhang N, Hu G, Myers TG, Williamson PR. Protocols for the Analysis of microRNA Expression, Biogenesis, and Function in Immune Cells. Curr Protoc Immunol. 2019;126:e78.

7. Bhaskaran M, Mohan M. MicroRNAs: history, biogenesis, and their evolving role in animal development and disease. Vet Pathol. 2014;51:759-74.

8. Saliminejad K, Khorram Khorshid HR, Soleymani Fard S, Ghaffari SH. An overview of microRNAs: Biology, functions, therapeutics, and analysis methods. J Cell Physiol. 2019;234:5451-65.

9. Zendjabil M, Favard S, Tse C, Abbou O, Hainque B. [The microRNAs as biomarkers: What prospects?]. C R Biol. 2017;340:114-31.

10. Zamanian Azodi M, Rezaei-Tavirani M, Rezaei-Tavirani M, Robati RM. Gestational Diabetes Mellitus Regulatory Network Identifies hsa-miR-145-5p and hsa-miR-875-5p as Potential Biomarkers. Int J Endocrinol Metab. 2019;17:e86640.

11. Ye L, Yu Y, Zhao Y. Icariin-induced miR-875-5p attenuates epithelial-mesenchymal transition by targeting hedgehog signaling in liver fibrosis. J Gastroenterol Hepatol. 2020;35:482-91.

12. Wang J, Lu Y, Ding H, Gu T, Gong C, Sun J, Zhang Z, Zhao Y, Ma C. The miR-875-5p inhibits SATB2 to promote the invasion of lung cancer cells. Gene. 2018;644:13-9.

13. Kang N, Ou Y, Wang G, Chen J, Li D, Zhan Q. miR-875-5p exerts tumor-promoting function via downregulation of CAPZA1 in esophageal squamous cell carcinoma. PeerJ. 2021;9:e10020.

14. Chen T, Sun L, Yao B, Wang L, Wang Y, Niu Y, Liu R, Mo H, Liu Z, Tu K, Liu Q. MicroRNA-875-5p inhibits tumor growth and metastasis of hepatocellular carcinoma by targeting eukaryotic translation initiation factor 3 subunit a. Oncol Rep. 2020;44:2067-79.

15. El Bezawy R, Cominetti D, Fenderico N, Zuco V, Beretta GL, Dugo M, Arrighetti N, Stucchi C, Rancati T, Valdagni $R$, et al. miR-875-5p counteracts epithelial-to-mesenchymal transition and enhances radiation response in prostate cancer through repression of the EGFR-ZEB1 axis. Cancer Lett. 2017;395:53-62. 
16. Zhang T, Cai X, Li Q, Xue P, Chen Z, Dong X, Xue Y. Hsa-miR-875-5p exerts tumor suppressor function through down-regulation of EGFR in colorectal carcinoma (CRC). Oncotarget. 2016;7:42225-40.

17. Tang $Y$, Meng X, Yu X, Shang H, Chen S, Liao L, Dong J. Inhibition of microRNA-875-5p promotes radioiodine uptake in poorly differentiated thyroid carcinoma cells by upregulating sodium-iodide symporter. J Endocrinol Invest. 2020;43:439-50.

18. Yang Z, Zhi Q, Wang D, Zhang L, Preston B, Brandon C, Kuang Y, Miao R, Shi Y, Guo X. Long Noncoding RNA C21orF96 Promotes the Migration, Invasion and Lymph Node Metastasis in Gastric Cancer. Anticancer Agents Med Chem. 2016;16:1101-8.

19. Carthew RW, Chodosh LA, Sharp PA. An RNA polymerase II transcription factor binds to an upstream element in the adenovirus major late promoter. Cell. 1985;43:439-48.

20. Miyamoto NG, Moncollin V, Egly JM, Chambon P. Specific interaction between a transcription factor and the upstream element of the adenovirus-2 major late promoter. Embo j. 1985;4:3563-70.

21. Sawadogo M, Van Dyke MW, Gregor PD, Roeder RG. Multiple forms of the human gene-specific transcription factor USF. I. Complete purification and identification of USF from HeLa cell nuclei. J Biol Chem. 1988;263:11985-93.

22. Gregor PD, Sawadogo M, Roeder RG. The adenovirus major late transcription factor USF is a member of the helix-loop-helix group of regulatory proteins and binds to DNA as a dimer. Genes Dev. 1990;4:1730-40.

23. Sirito M, Lin Q, Deng JM, Behringer RR, Sawadogo M. Overlapping roles and asymmetrical crossregulation of the USF proteins in mice. Proc Natl Acad Sci U S A. 1998;95:3758-63.

24. Yago M, Ohki R, Hatakeyama S, Fujita T, Ishikawa F. Variant forms of upstream stimulatory factors (USFs) control the promoter activity of hTERT, the human gene encoding the catalytic subunit of telomerase. FEBS Lett. 2002;520:40-6.

25. Yan S, Sloane BF. Isolation of a novel USF2 isoform: repressor of cathepsin B expression. Gene. 2004;337:199-206.

26. Sirito M, Lin Q, Maity T, Sawadogo M. Ubiquitous expression of the 43- and 44-kDa forms of transcription factor USF in mammalian cells. Nucleic Acids Res. 1994;22:427-33.

27. Roth U, Jungermann K, Kietzmann T. Modulation of glucokinase expression by hypoxia-inducible factor 1 and upstream stimulatory factor 2 in primary rat hepatocytes. Biol Chem. 2004;385:239-47.

28. Bruno MEC, West RB, Schneeman TA, Bresnick EH, Kaetzel CS. Upstream stimulatory factor but not cMyc enhances transcription of the human polymeric immunoglobulin receptor gene. Mol Immunol. 2004;40:695-708.

29. Ocejo-Garcia M, Baokbah TA, Ashurst HL, Cowlishaw D, Soomro I, Coulson JM, Woll PJ. Roles for USF-2 in lung cancer proliferation and bronchial carcinogenesis. J Pathol. 2005;206:151-9.

30. Tan Y, Chen Y, Du M, Peng Z, Xie P. USF2 inhibits the transcriptional activity of Smurf1 and Smurf2 to promote breast cancer tumorigenesis. Cell Signal. 2019;53:49-58. 
31. Chi TF, Horbach T, Gotz C, Kietzmann T, Dimova EY: Cyclin-Dependent Kinase 5 (CDK5)-Mediated Phosphorylation of Upstream Stimulatory Factor 2 (USF2) Contributes to Carcinogenesis. Cancers (Basel) 2019, 11.

32. Christensen LL, Tobiasen H, Holm A, Schepeler T, Ostenfeld MS, Thorsen K, Rasmussen MH, Birkenkamp-Demtroeder K, Sieber OM, Gibbs P, et al. MiRNA-362-3p induces cell cycle arrest through targeting of E2F1, USF2 and PTPN1 and is associated with recurrence of colorectal cancer. Int J Cancer. 2013;133:67-78.

33. Lee YS, Dutta A. MicroRNAs in cancer. Annu Rev Pathol. 2009;4:199-227.

34. Kwan JY, Psarianos P, Bruce JP, Yip KW, Liu FF. The complexity of microRNAs in human cancer. J Radiat Res. 2016;57(Suppl 1):i106-11.

35. Tong F, Cao P, Yin Y, Xia S, Lai R, Liu S. MicroRNAs in gastric cancer: from benchtop to bedside. Dig Dis Sci. 2014;59:24-30.

36. Hayes J, Peruzzi PP, Lawler S. MicroRNAs in cancer: biomarkers, functions and therapy. Trends Mol Med. 2014;20:460-9.

37. Bertoli G, Cava C, Castiglioni I. MicroRNAs: New Biomarkers for Diagnosis, Prognosis, Therapy Prediction and Therapeutic Tools for Breast Cancer. Theranostics. 2015;5:1122-43.

38. Xing X, Du X, Lu Z, Ning T, Su X, Ke Y. Characterization of the promoter of 1A6/DRIM, a novel cancerrelated gene and identification of its transcriptional activator. Gene. 2005;344:161-9.

39. Horbach T, Gotz C, Kietzmann T, Dimova EY. Protein kinases as switches for the function of upstream stimulatory factors: implications for tissue injury and cancer. Front Pharmacol. 2015;6:3.

40. Choe C, Chen N, Sawadogo M. Decreased tumorigenicity of c-Myc-transformed fibroblasts expressing active USF2. Exp Cell Res. 2005;302:1-10.

41. Chen N, Szentirmay MN, Pawar SA, Sirito M, Wang J, Wang Z, Zhai Q, Yang HX, Peehl DM, Ware JL, Sawadogo M. Tumor-suppression function of transcription factor USF2 in prostate carcinogenesis. Oncogene. 2006;25:579-87.

42. Xie F, Ling L, van Dam H, Zhou F, Zhang L. TGF- $\beta$ signaling in cancer metastasis. Acta Biochim Biophys Sin (Shanghai). 2018;50:121-32.

43. Syed V. TGF- $\beta$ Signaling in Cancer. J Cell Biochem. 2016;117:1279-87.

44. Taniguchi S, Elhance A, Van Duzer A, Kumar S, Leitenberger JJ, Oshimori N. Tumor-initiating cells establish an IL-33-TGF-beta niche signaling loop to promote cancer progression. Science 2020, 369.

45. Li Q, Yue W, Li M, Jiang Z, Hou Z, Liu W, Ma N, Gan W, Li Y, Zhou T, et al. Downregulating Long Noncoding RNAs CTBP1-AS2 Inhibits Colorectal Cancer Development by Modulating the miR-93-5p/TGFbeta/SMAD2/3 Pathway. Front Oncol. 2021;11:626620.

\section{Figures}



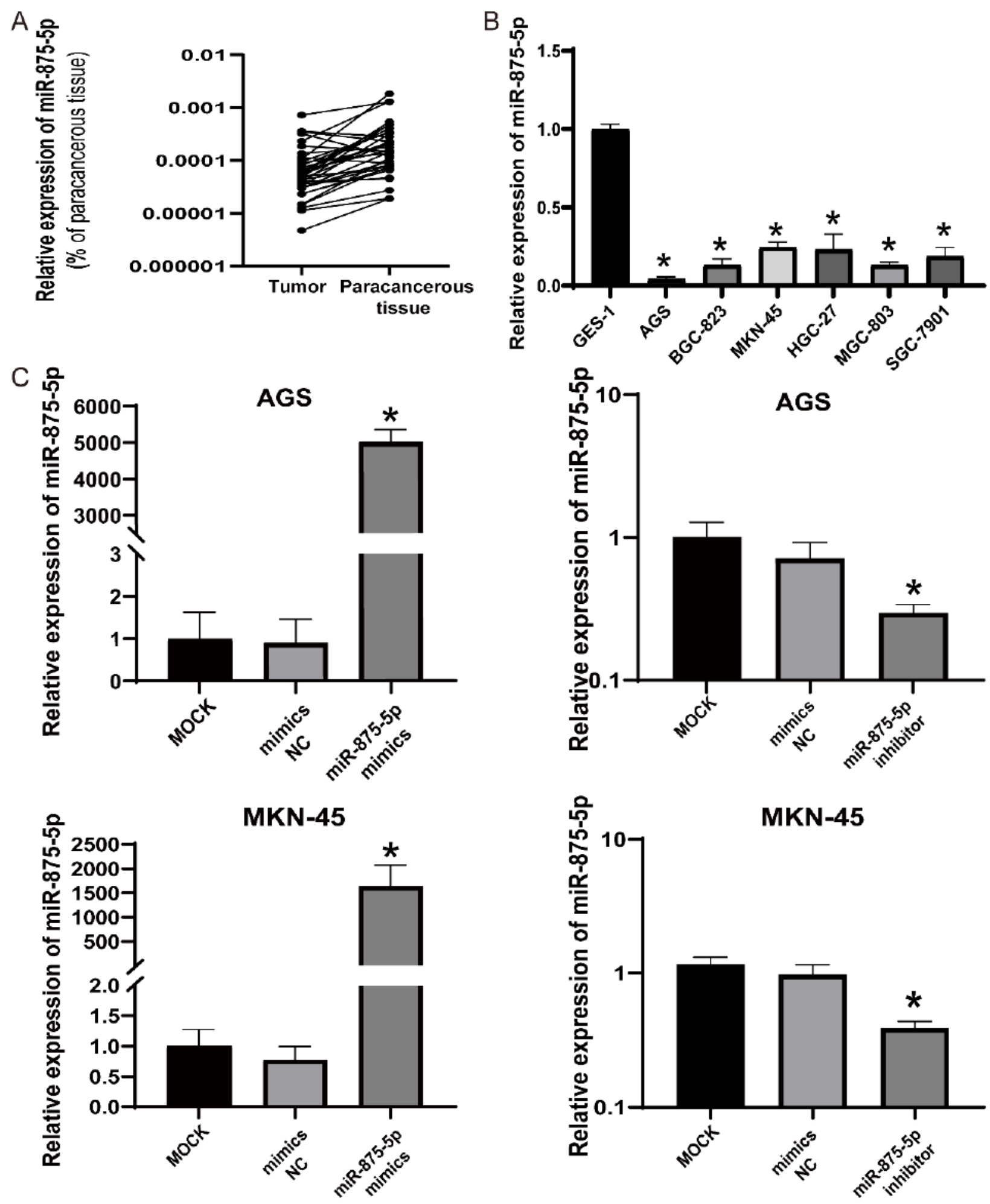

Figure 1

Expression of miR-875-5p in GC tissues, GC cells and transfected cells. (A) The expression levels of miR875-5p in 28 pairs of human GC tissues and adjacent normal tissues were detected by miRNA RT-PCR. (B) The expression level of miR-875-5p in GC cells and GES-1 was examined by miRNA RT-PCR. (C) The efficacy of transfection was verified in cells transfected with miR-875-mimics and miR-875-inhibitor. * $\mathrm{P}<$ 0.05. Data expressed as mean $\pm S D$. 

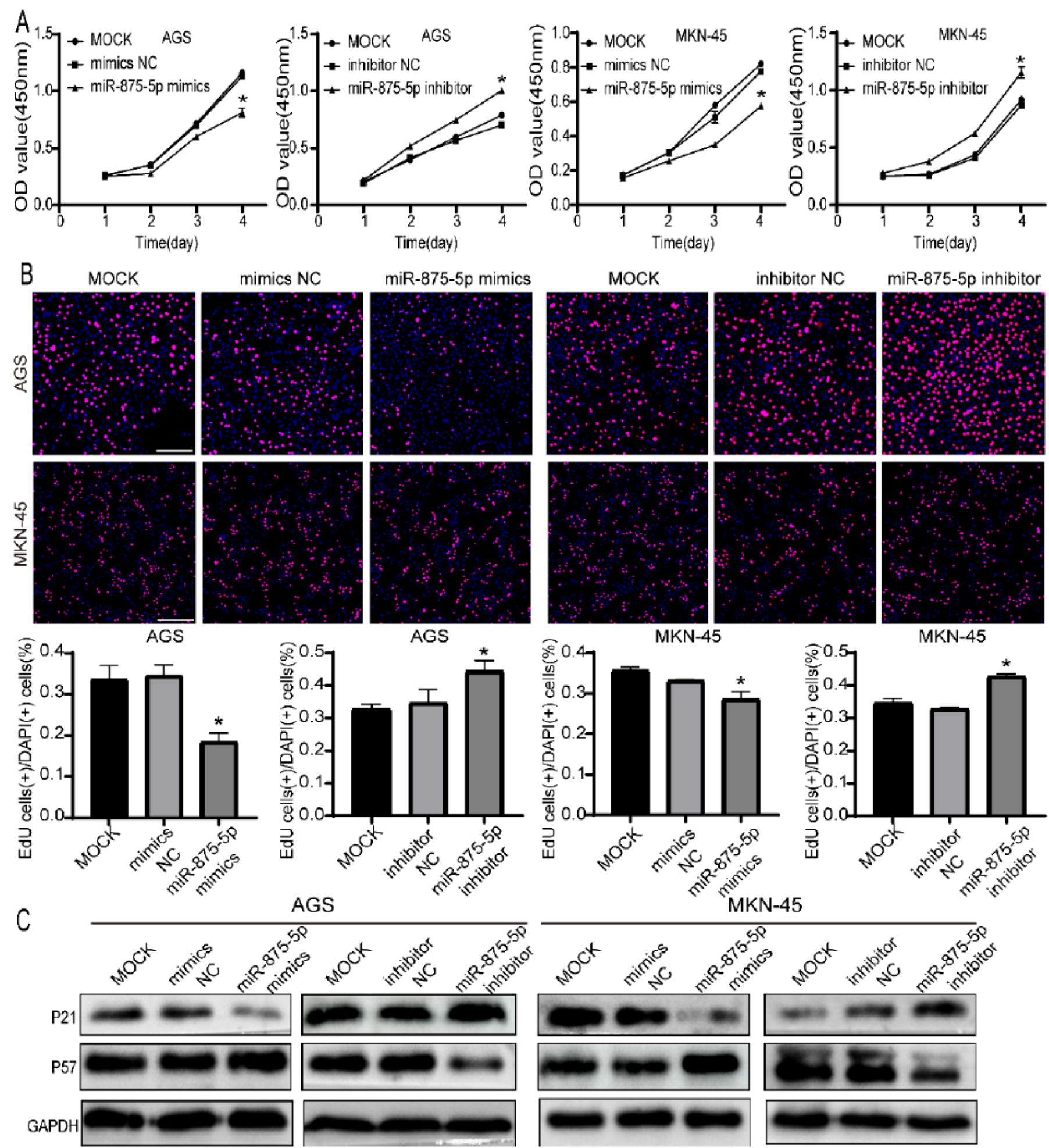

AGS
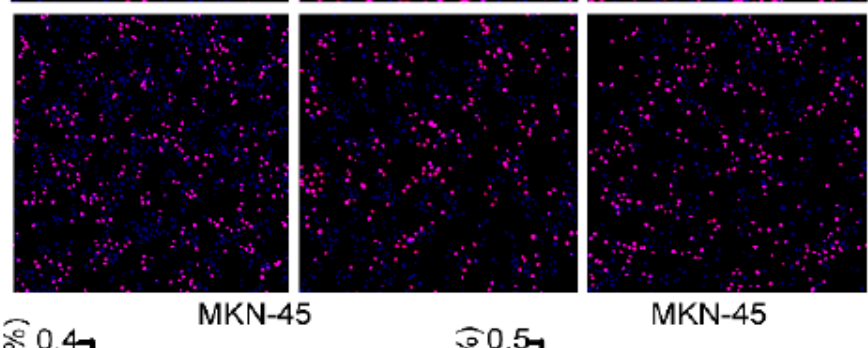

MKN-45
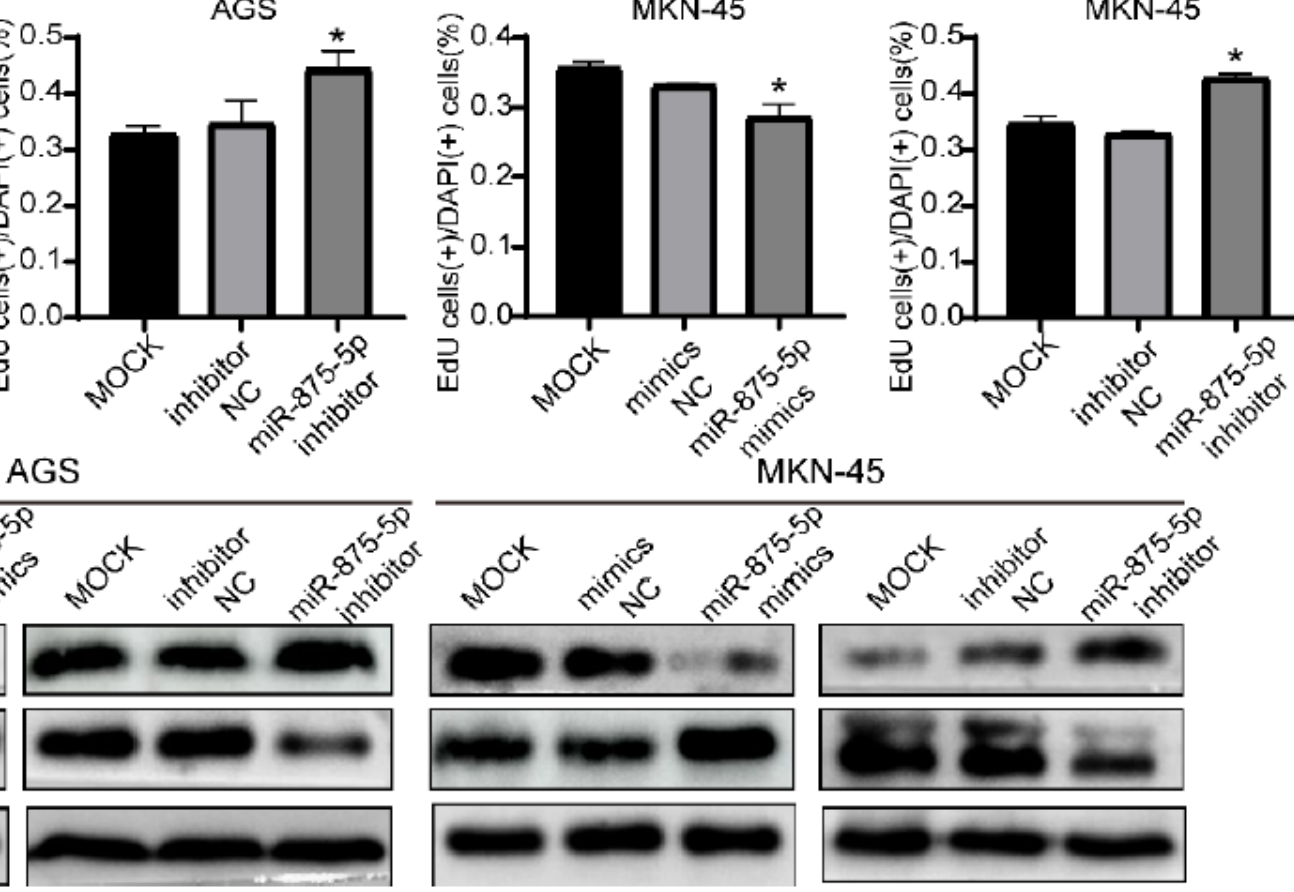

Figure 2

miR-875-5p inhibited the proliferation of GC cells in vitro. (A) CCK8 assay was used to evaluate the effect of miR-875-5p on the proliferation of AGS or MKN-45 cells. (B) EDU assay showed the effect of overexpression or silencing of miR-875-5p on the growth of GC cells. (C) Western blotting showed the levels of proliferation-related proteins (P21 and P57) in miR-875-5p overexpressed and miR-875-5p silenced AGS or MKN-45 cells and their negative control cells. * $P<0.05$. Data expressed as mean \pm SD. 
Figure 3

miR-875-5p inhibited migration and invasion in vitro and induced associated protein changes.
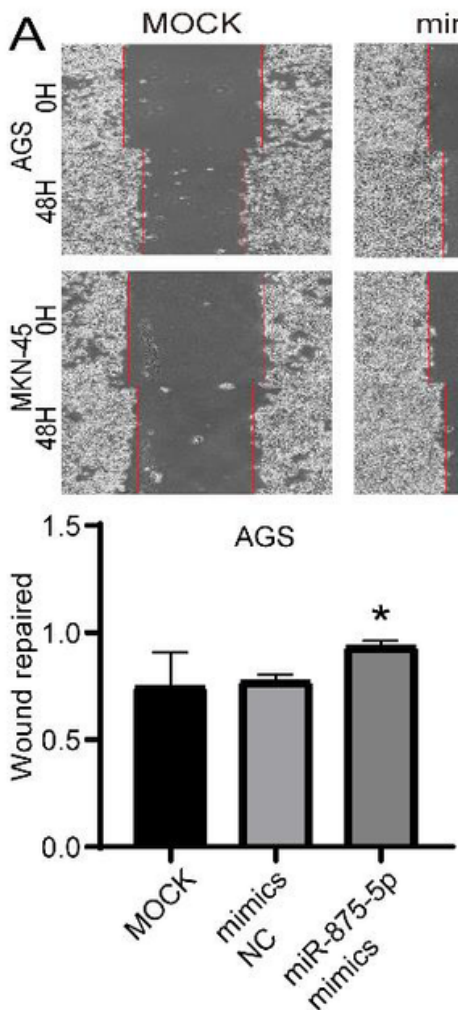

mimics NC
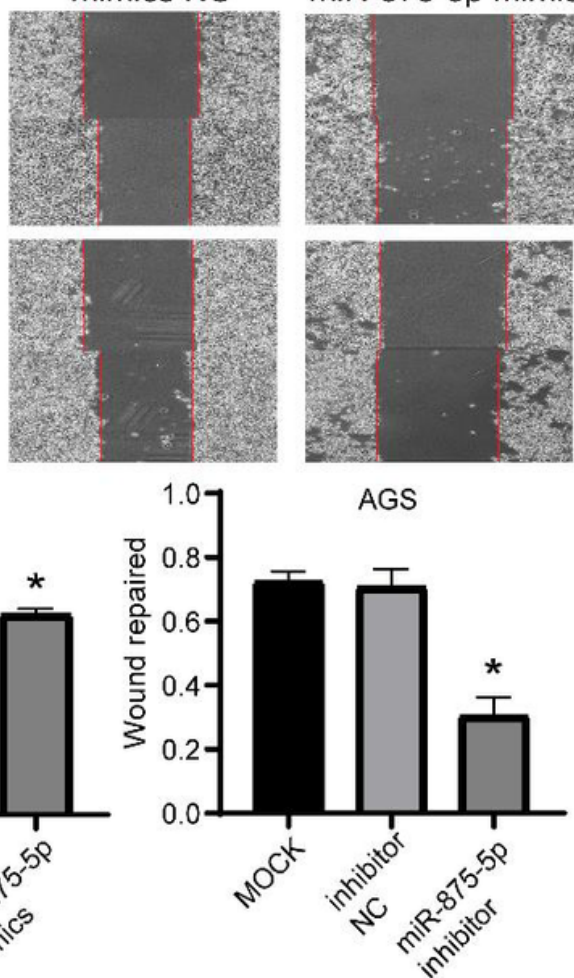
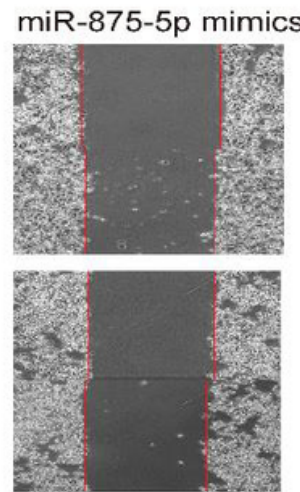

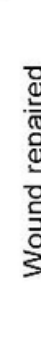
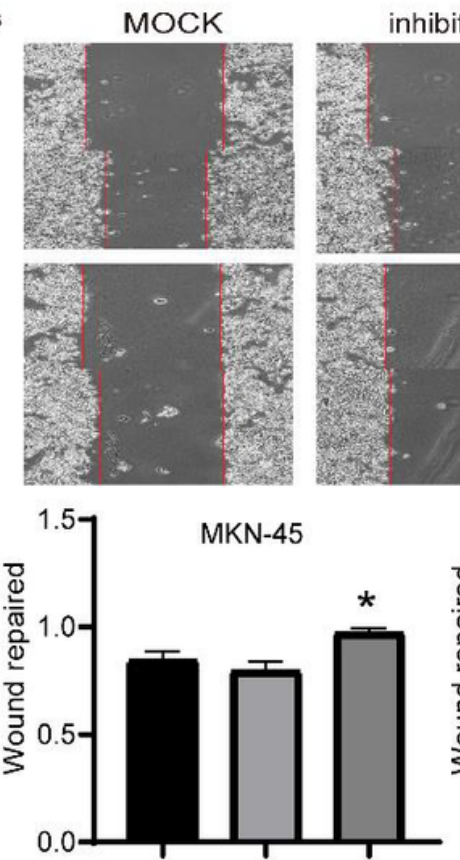

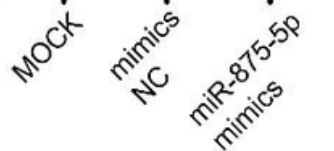

inhibitor NC
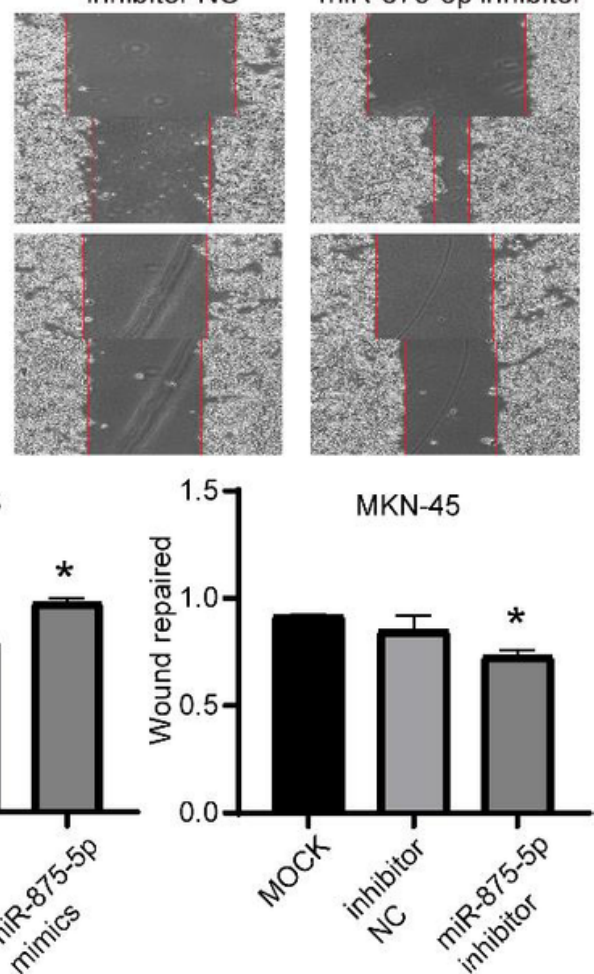

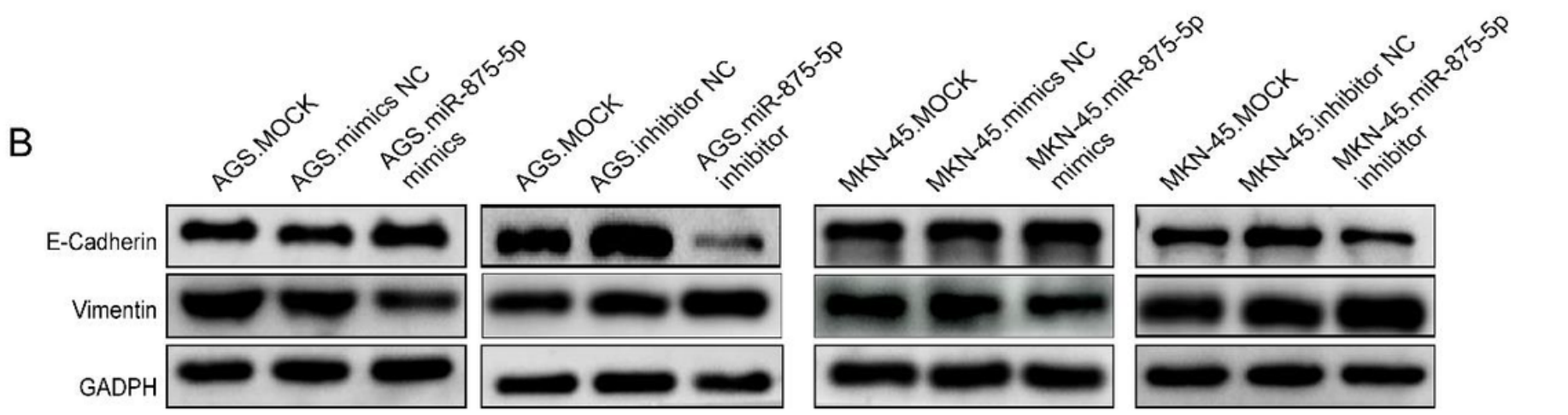

Figure 4

miR-875-5p inhibited migration and invasion in vitro and induced associated protein changes. (A) The wound healing test was performed to study the changes of miR-875-5p overexpression and silencing on the migration ability of GC cells. The percentage of wound closure or the number of transmembrane cells were calculated and compared. (B) Western blot analysis showed that miR-875-5p overexpression and or silencing increased or decreased E-cadherin and Vimentin protein expression, respectively. ${ }^{*}<<0.05$. Data expressed as mean $\pm S D$. 


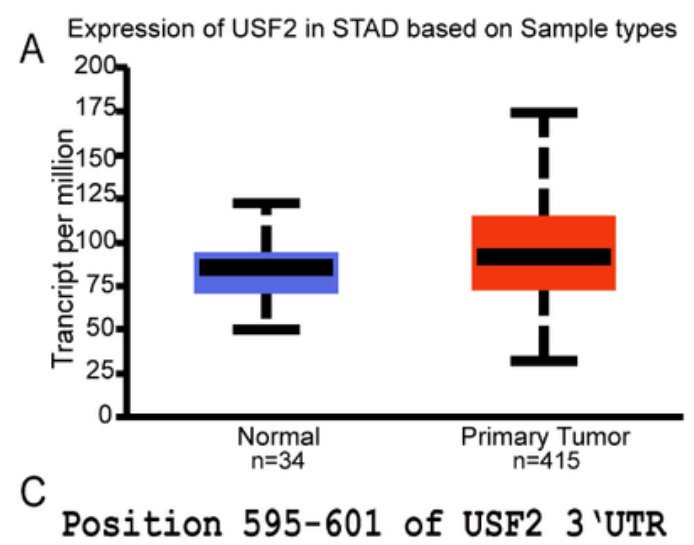

has-miR-875-5p

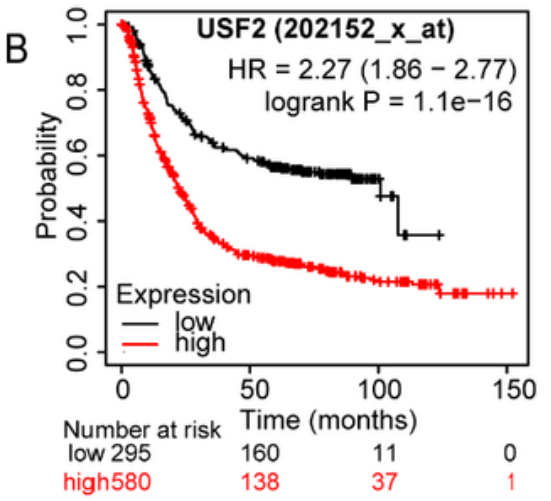

: 5 ' aaaaaAAUAGAGAGAGAGGUAUu 3'

III |:| ||||||||

: 3 ' guggacUAU-UUUGACUCCAUAu 5 '

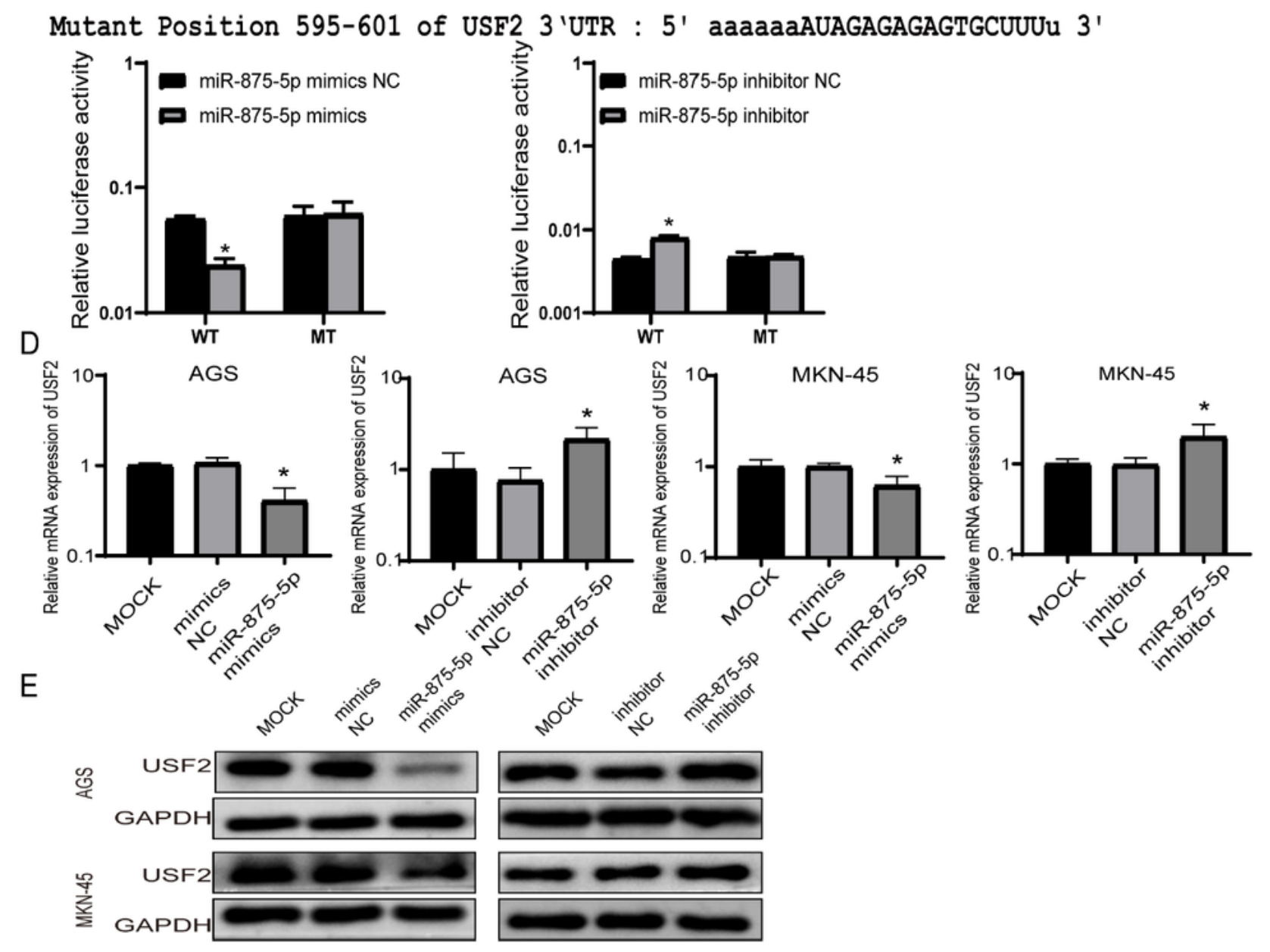

Figure 5

USF2 was up-regulated in GC tissues and confirmed to be a direct target gene of miR-875-5p. (A) Expression of USF2 in GC and normal gastric tissue. (B) Overall survival curve of USF2 expression in GC patients (C) Luciferase reporter assay was performed to confirm that miR-875-5p directly bound to the 3'UTR region of USF2. The results of luciferase activity were analyzed in cells treated with miR-875-5pmimics or negative control and miR-875-5p-inhibitor or negative control. (D) The expression level of USF2 
mRNA in AGS and MKN-45 cells after transfected was detected by qRT-PCR. (E) The expression level of USF2 protein in AGS and MKN-45 cells after transfection was verified by Western blot. * $P<0.05$. Data expressed as mean $\pm S D$.
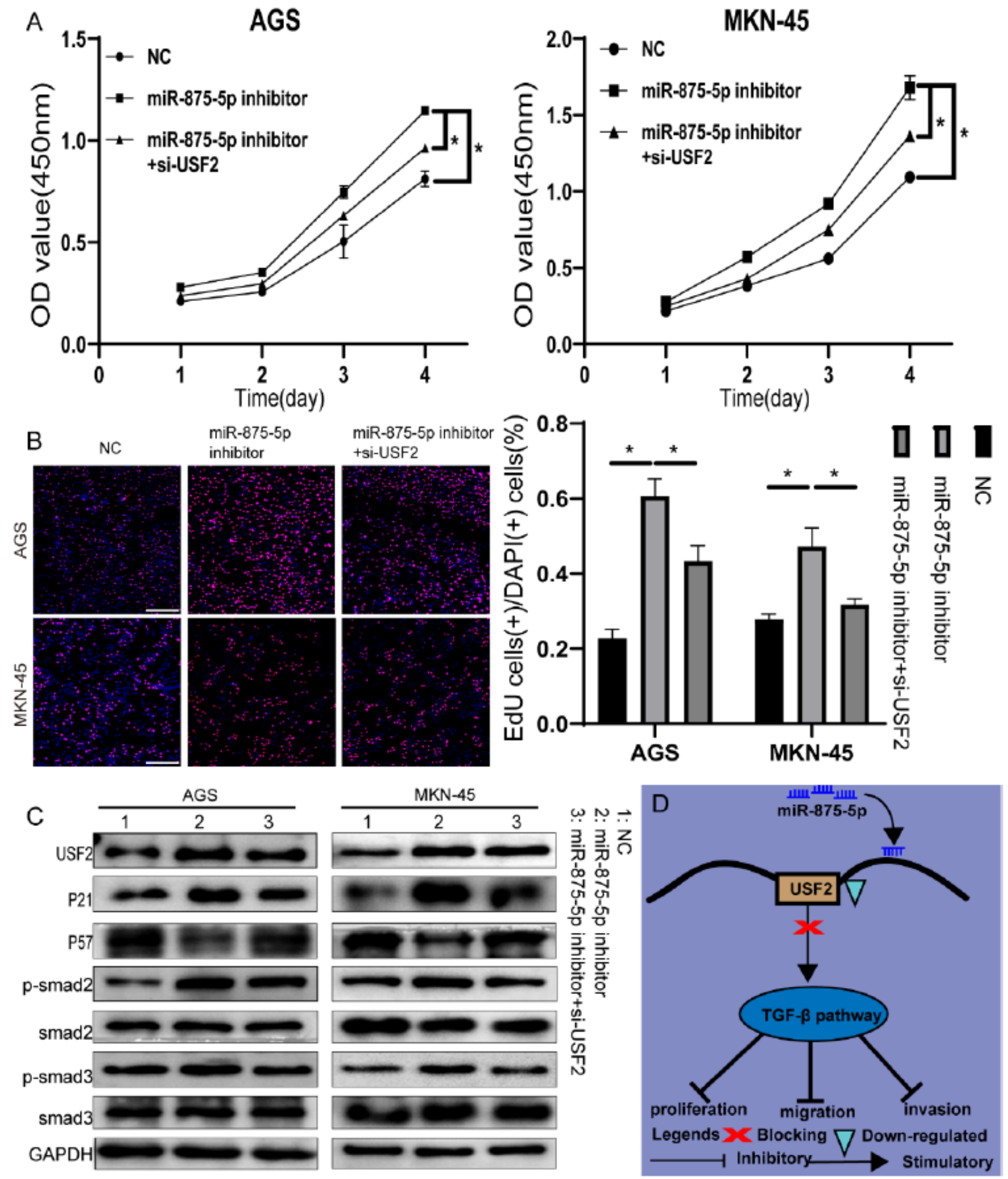

Figure 6

The effect of miR-875-5p silencing on GC cells was partially offset by USF2 knockdown. (A) CCK8 assay was performed to determine that USF2 knockdown could partly counteract miR-875-5p silencing-induced 
proliferation in MKN-45 and AGS cells. and (B) EDU assay was performed to determine that USF2 knockdown could partly counteract miR-875-5p silencing-induced proliferation in MKN-45 and AGS cells. (C) Western blot showed the changes of related proteins such as USF2, P21, P57, Smad2, p-Smad2, Smad3 and p-Smad3 in AGS and MKN-45 cells after transfection. (D) Proposed model of miR-875-5p regulating USF2 and affecting TGF- $\beta$ signaling pathway. ${ }^{*} P<0.05$; Data expressed as mean \pm SD.
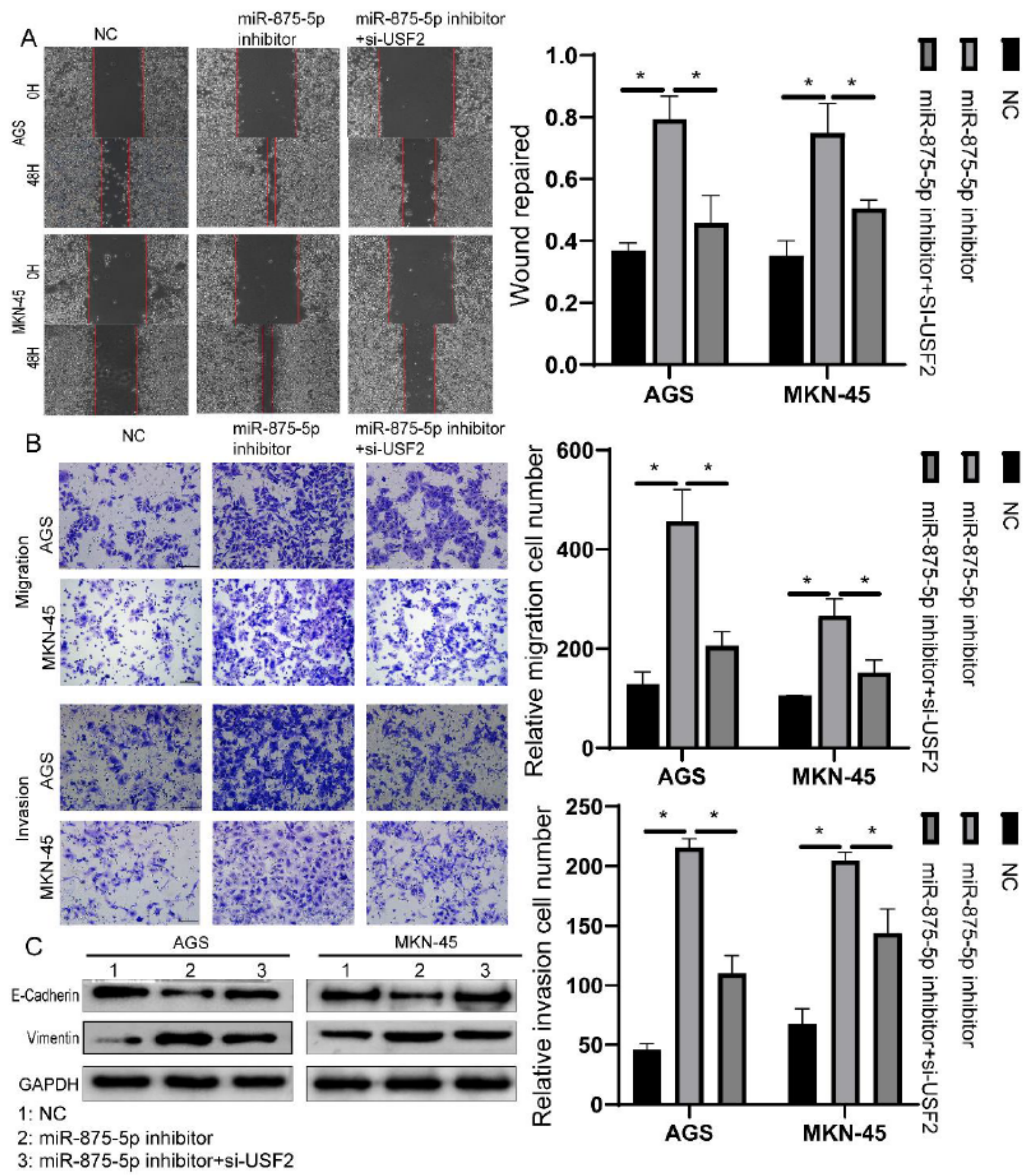

Figure 7 
The effect of miR-875-5p silencing on GC cells was partially offset by USF2 knockdown (A) Changes in cell migration were examined by wound healing assayed in AGS cells and MKN-45 cells. (B) Transwell assay was used to confirm the influence of USF2 alterations on GC cell migration and invasion. (C) Western blot showed the changes of related proteins such as E-Cadherin and Vimentin in AGS and MKN45 cells after transfection. ${ }^{*} P<0.05$; Data expressed as mean $\pm S D$.

\section{Figure 8}

miR-875-5p inhibits tumor formation in vivo. (A) xenograft tumors were obtained from different groups of nude mice injected with MKN-45 cells transfected with miR-875-5p NC and miR-875-5p-mimics, respectively. (B) The expression of USF2 in the xenograft tumors was determined by immunohistochemistry. (C) The tumor growth curve and tumor weight were significantly different between miR-875-5p mimics group and miR-875-5p NC group. * $\mathrm{P}<0.05$; Data expressed as mean \pm SD.

\section{Supplementary Files}

This is a list of supplementary files associated with this preprint. Click to download.

- figs9.png

- fids10.png 